\title{
Midthoracic Pain, Sentinel Arterial Haemorrhage and Exsanguination after a Symptom-Free Interval (Chiari's Triad) is Diagnostic of Arterio- Oesophageal Fistula: A Life-Threatening Cause of Gastrointestinal Bleeding
}

\author{
Andra Glodean ${ }^{1}$, Rainer Grobholz ${ }^{2,3}$, Karim El-Hag ${ }^{1}$, Mairi Ziaka ${ }^{1}$, Jean-Paul Schmid ${ }^{1,4}$ \\ ${ }^{1}$ Department of Internal Medicine, Clinic Barmelweid, Barmelweid, Switzerland \\ ${ }^{2}$ Institute of Pathology, Cantonal Hospital Aarau, Aarau, Switzerland \\ ${ }^{3}$ Medical Faculty, University of Zurich, Zurich, Switzerland \\ ${ }^{4}$ Medical Faculty, University of Bern, Bern, Switzerland
}

\section{Doi: 10.12890/2021_002134- European Journal of Case Reports in Internal Medicine - ( ) EFIM 2021}

Received: 05/01/2021

Accepted: $31 / 01 / 2021$

Published: $23 / 02 / 2021$

\begin{abstract}
How to cite this article: Glodean A, Grobholz R, El-Hag K, Ziaka M, Schmid JP. Midthoracic pain, sentinel arterial haemorrhage and exsanguination after a symptom-free interval (chiari's triad) is diagnostic of arterio-oesophageal fistula: a life-threatening cause of gastrointestinal bleeding. EJCRIM 2021;8: doi:10.12890/2021_002134.
\end{abstract}

Conflicts of Interests: The Authors declare that there are no competing interests.

This article is licensed under a Commons Attribution Non-Commercial 4.0 License

\section{ABSTRACT}

Introduction: Arterio-oesophageal fistulae are a very uncommon cause of severe gastrointestinal bleeding, and mostly result from an aberrant right subclavian artery and mediastinal surgery or prolonged endotracheal/nasogastric intubation.

Material and Methods: We present the case of a patient with an oesophageal adenocarcinoma and haematemesis due to a subclavian arteriooesophageal fistula after mediastinal radiotherapy.

Conclusion: We discuss the rare, life-threatening condition of acute erosion of the left subclavian artery caused by an oesophageal tumour and presenting with Chiari's triad.

\section{LEARNING POINTS}

- Subclavian arterio-oesophageal fistula is an uncommon, life-threatening cause of gastrointestinal bleeding.

- Knowledge of Chiari's triad enables early recognition of potentially fatal gastro-intestinal bleeding.

- A high level of suspicion is essential for prompt diagnosis and referral for surgical treatment especially in patients with malignancies of the upper gastrointestinal tract.

\section{KEYWORDS}

Subclavian arterio-oesophageal fistula, oesophageal carcinoma, gastrointestinal bleeding, Chiari's triad, exsanguination, sentinel arterial haemorrhage

\section{CASE DESCRIPTION}

A 68-year-old man was referred to the internal medicine department of an external hospital with a 6-month history of thoracic spine pain that was radiating to the trapezius and levator scapulae muscles. The patient noted a significant involuntary weight loss and had developed signs of Horner's syndrome. His history included smoking (50 pack-years) and hypercholesterolaemia. The patient was on atorvastatin 40 $\mathrm{mg} /$ day, aspirin $100 \mathrm{mg} /$ day and bisoprolol $5 \mathrm{mg} /$ day. 
On otorhinolaryngological examination, left-sided recurrent nerve paresis was diagnosed and a neck/chest computed tomography (CT) scan detected a mediastinal tumour between the left common carotid and left subclavian artery ( $33 \times 22 \mathrm{~mm})$ in close contact with the second thoracic vertebral body (Fig. 1). Multiple pulmonary nodules (up to $4 \mathrm{~mm}$ ) as well as a left adrenal gland tumour (27×35 mm) were also present.

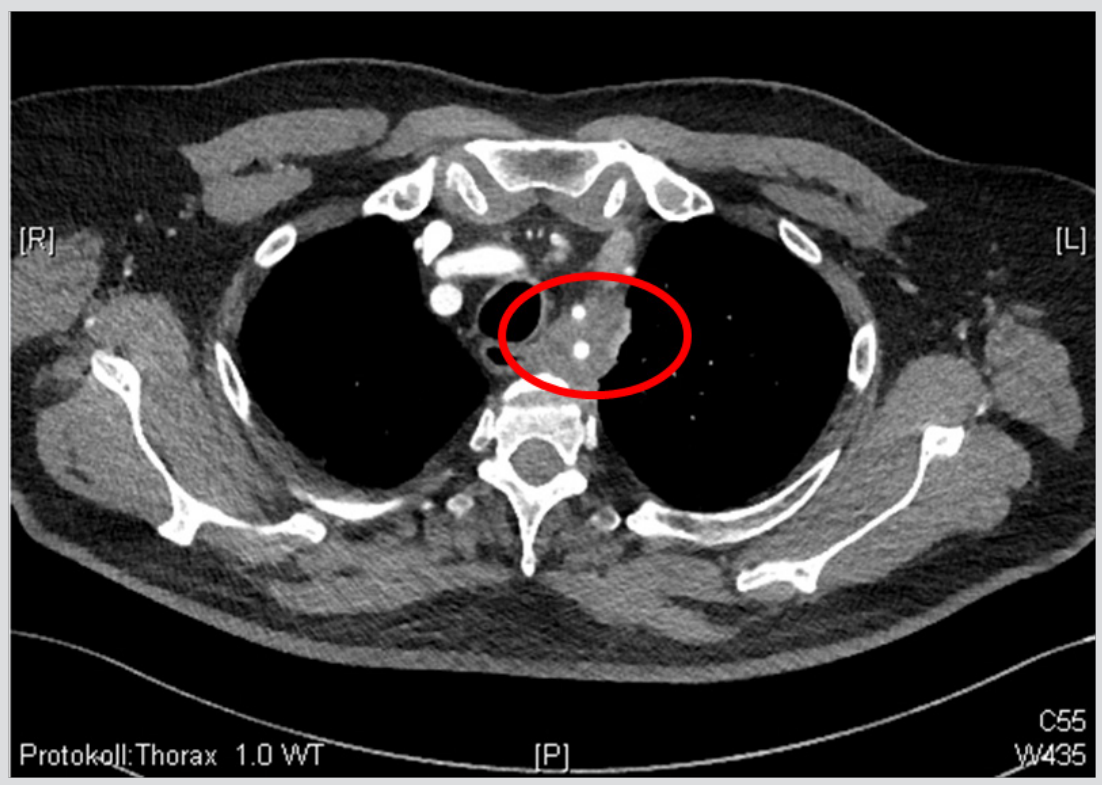

Figure 1. CT scan showing a mediastinal tumour (red circle) with the left subclavian artery passing centrally through it

Bronchoscopy with endoscopic ultrasound-guided fine-needle aspiration biopsy of the mediastinal tumour revealed an adenocarcinoma. PET-CT was performed for further staging and showed a metabolically highly active tumour with the left subclavian artery passing centrally through it. The patient underwent diagnostic thoracoscopy with mediastinal biopsy and wedge resection of the left upper pulmonary lobe. The mediastinal biopsies showed a poorly differentiated adenocarcinoma and the final diagnosis of a poorly differentiated oligometastatic lung adenocarcinoma cT2b cNO cM1c stage IVb was made.

Due to the local tumour extension and the arterial involvement, surgery was not carried out but combined mediastinal radiotherapy $(30 \times 200$ cGy) and chemotherapy (cisplatin and etoposide), followed by maintenance therapy with the programmed cell death ligand 1-antibody durvalumab together with stereotactic radiotherapy of the adrenal metastasis, were planned.

After completion of mediastinal radio- and chemotherapy, the patient was admitted to our institution for rehabilitation. He presented in a reduced, asthenic general condition and complained of dysgeusia, loss of appetite and nausea. Due to a stricture of the oesophagus with persistent dysphagia, enteral nutrition was maintained through a percutaneous endoscopic gastrostomy tube. His medication was supplemented with esomeprazole $20 \mathrm{mg} /$ day and enoxaparin $5000 \mathrm{IU} /$ day s.c. as thromboprophylaxis.

The patient was integrated into the multidisciplinary rehabilitation program and the course was uneventful until day 25 of hospitalization, when he complained of exacerbation of a left thoracic pain radiating to the left shoulder, which had occurred for the first time 2 weeks previously and initially responded well to the administration of analgesics. Over the next 2 days, the intensity of pain increased with radiation to the spine. On day 27 of hospitalization, the patient developed two episodes of haematemesis with syncope and hypotension. Haemodynamic stabilization measures were immediately applied and the patient was transferred to the referring hospital, where haemorrhagic shock progressed due to repeated severe haematemesis. Haemodynamic stabilization was unsuccessful and the patient developed progressive hypoxaemia due to blood aspiration and died of uncontrollable haemorrhagic shock and hypoxia.

The autopsy revealed an $8 \mathrm{~mm}$ perforated ulcer in the upper third of the oesophageal wall (Fig. 2) originating from a $4.0 \times 3.5 \times 2.0 \mathrm{~cm}$ regressed mediastinal tumour. Metastases were present in both adrenal glands and the right kidney.

The source of bleeding was identified as a fistula between the left subclavian artery and the oesophageal ulcer due to infiltration by the tumour. Treatment-related necrotic changes were also detected (Fig. 3). 


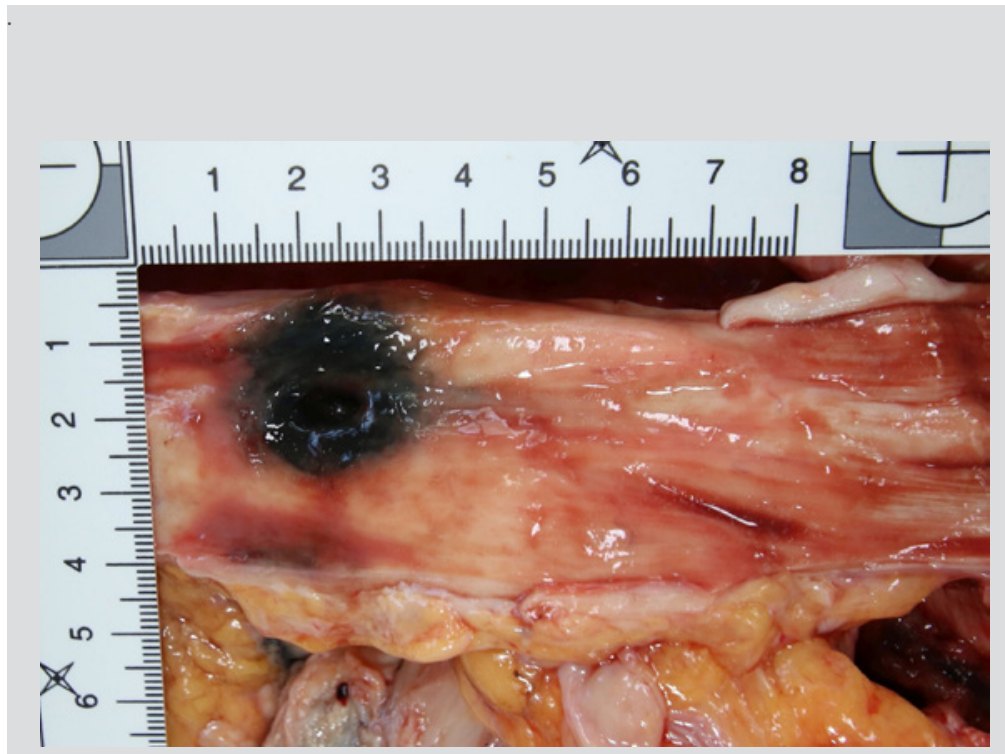

Figure 2. Perforated ulcer in the upper third of the oesophageal wall with the mediastinal tumour lying underneath. The surrounding mucosa is haemorrhagic due to massive bleeding

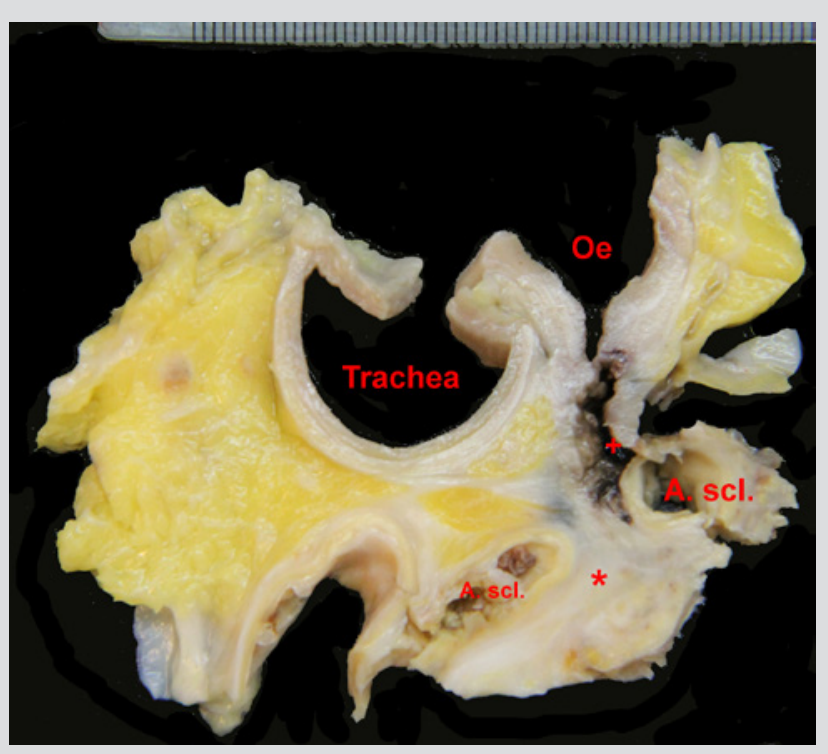

Figure 3. Cross-section through the tumour, oesophagus, trachea and subclavian artery with a fistula between the oesophagus (Oe) and the subclavian artery (A. scl.). The tumour appears white $\left({ }^{*}\right)$ and the fistula is marked (+)

\section{DISCUSSION}

The most common causes of haematemesis include peptic ulcer, oesophageal and gastric varices, erosive oesophagitis or vascular lesions. Malignancy of the upper gastrointestinal tract is responsible for less than $3 \%$ of severe bleeding events, usually resulting from erosion into an underlying vessel. Upper gastrointestinal bleeding caused by an aorto-enteric fistula is rare, aortic aneurysms being the most common risk factor, followed by a history of aortic surgery and oesophageal/bronchial malignancy ${ }^{[1]}$.

Left subclavian artery-oesophageal fistula are very uncommon and described mostly in relation to the ingestion of fish bones. Only a few cases of aorto-oesophageal fistulae due to an ulcerated oesophageal neoplasm without previous surgery have been published. To the best of our knowledge, this is the first report of a patient with a left subclavian artery-oesophageal fistula without a pre-existing anomaly or surgery. Due to the fact that the last imaging control with CT of the thorax showed regression of the tumour without additional abnormalities and gastroscopy showed oesophageal stenosis without histopathological evidence of malignancy, the occurrence of a complication was very much unexpected.

However, unexplained chest pain in patients with known malignancies of the upper gastrointestinal tract should raise suspicion of an imminent complication.

Chest pain or dysphagia, sentinel arterial haemorrhage, followed by a symptom-free interval of hours to days, and finally exsanguination due to massive haemorrhage, are signs known as Chiari's triad ${ }^{[2]}$. Spontaneous rupture of the aorta, or as in our case of the left subclavian artery through an aorto- or arterio-oesophageal fistula into a closely adherent portion of the gastrointestinal tract, may lead to gastrointestinal bleeding, which initially may be only minor and intermittent, due to the formation of thrombus plugging the fistula as a result of hypotension (the so-called sentinel or herald bleeding). However, the plug may be pushed out of its canal, leading to further bleeding after the patient becomes normotensive and result in exsanguination. Sentinel haemorrhage may occur in a repetitive fashion, the time interval between the initial haemorrhage and final exsanguination ranging from hours to months ${ }^{[3]}$.

A review of cases of aorto-oesophageal fistulae over a 30-year period showed midthoracic pain was present in $59 \%$, dysphagia in $45 \%$ and a sentinel haemorrhage in $65 \%$ of patients. Overall, $45 \%$ of patients met all the criteria of Chiari's triad ${ }^{[3,4]}$.

The literature also highlights the potential high risk of fistulae developing in patients with oesophageal carcinoma undergoing radio- or chemotherapy ${ }^{[5,6]}$. In our case, histology showed radio- and chemotherapy-induced regression with necrosis along with inflammatoryresorptive changes inside the tumour and the adjacent oesophagus, which finally resulted in a tumourous/inflammatory erosion of the subclavian artery. Interestingly, significant regression of the mediastinal tumour with a minimal residual vital component (<1\%) was observed. The post-mortem examination strongly suggested tumour infiltration as the primary cause of the fistula, but radiation has also been reported to have an impact on the development of aorto-oesophageal fistulae ${ }^{[7]}$. 


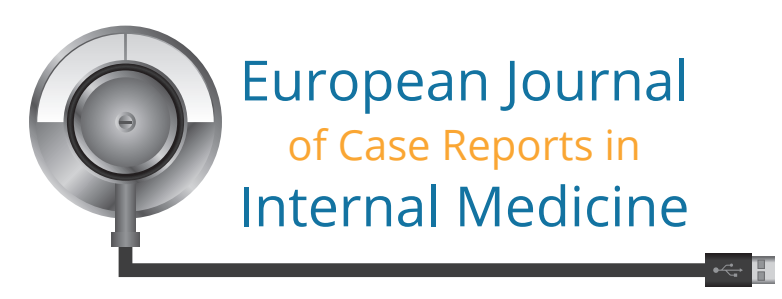

The insertion of a Blakemore tube could have provided time for additional diagnostic and eventually therapeutic procedures. Surgery for bleeding control and repair of the arterial wall would have been the treatment of choice, although an endovascular approach with placement of a stent graft in the left subclavian artery or transcatheter arterial embolization would have been alternative strategies, even though only as a temporary measure.

In conclusion, the diagnosis of an arterio-oesophageal fistula is difficult because of its rarity and unspecific presentation. However, knowledge of Chiari's triad allows physicians to have a high level of suspicion at the time of initial bleeding and timely referral for life-saving surgery.

\section{REFERENCES}

1. Kamboj AK, Hoversten P, Leggett CL. Upper gastrointestinal bleeding: etiologies and management. Mayo Clin Proc 2019;94(4):697-703

2. Chiari H. Ueber Fremdkoerperverletzung des Oesophagus mit Aortenperforation. Berl Klin Wochenschr 1914;51:7.

3. Saers SJ, Scheltinga MR. Primary aortoenteric fistula. Br J Surg 2005;92(2):143-152.

4. Hollander JE, Quick G. Aortoesophageal fistula: a comprehensive review of the literature. Am J Med 1991;91(3):279-287.

5. Kawakami T, Tsushima T, Omae K, Ogawa H, Shirasu H, Kito Y, et al. Risk factors for esophageal fistula in thoracic esophageal squamous cell carcinoma invading adjacent organs treated with definitive chemoradiotherapy: a monocentric case-control study. BMC Cancer 2018;18(1):573.

6. Taniguchi H, Yamazaki K, Boku N, Funakoshi T, Hamauchi S, Tsushima T, et al. Risk factors and clinical courses of chemoradiation-related arterio-esophageal fistula in esophageal cancer patients with clinical invasion of the aorta. Int J Clin Oncol 2011;16(4):359-365.

7. Sivaraman SK, Drummond R. Radiation-induced aortoesophageal fistula: an unusual case of massive upper gastrointestinal bleeding. J Emerg Med 2002;23(2):175-178. 